\title{
DIAGNOSTICS OF THE STOCHASTIC IONOSPHERIC CHANNEL IN THE DECAMETER BAND OF RADIO WAVES
}

\author{
N.T. Afanasiev \\ Irkutsk State University, \\ Irkutsk, Russia, spacemaklay@gmail.com
}

\author{
S.O. Chudaev \\ Institute of Solar-Terrestrial Physics SB RAS, \\ Irkutsk, Russia,ch45st@gmail.com
}

\begin{abstract}
We propose a method for direct diagnostics of a stochastic ionospheric radio channel. This method can recalculate probe signal characteristics into transmitted signal characteristics. We derive analytical equations of second-order statistical moments for trajectory characteristics of the main and probe signals propagating in a three-dimensional randomly inhomogeneous ionosphere. We take into account boundary conditions at signal transmission and reception points. As a model of random irregularities of permittivity of the ionosphere, we utilize the concept of a changing space-time correlation ellipsoid, which is self-consistent with spatial changes in the middle ionosphere. Time fluctuations of random irregularities are taken into account by the hypothesis of frozen transfer. We use analytical relationships to calculate the expected statistical characteristics of decameter signals along oblique probing paths of the ionosphere. An operational numerical algorithmization of the formulas derived is proposed. We report re-
\end{abstract}

sults of numerical experiments to determine the expected phase variances, group delay, and Doppler frequency shift of the main signal on a given single-hop path, based on measurements of these characteristics of a probe signal on a secondary path. We demonstrate the efficiency of the proposed method for diagnosing statistical trajectory characteristics of a decameter signal along single-hop paths under conditions when ground points of transmission and reception of the main and probe signals are outside the vicinity of focusing points of the wave field.

Keywords: ionosphere, random irregularities, fluctuations, statistical moments, ray approximation, radio signal, decameter band.

\section{INTRODUCTION}

Ensuring the reliability and noise immunity of the ionospheric radio channel requires a priori information about its physical parameters and properties [Blagoveshchensky, Zherebtsov, 1987; Rawer, 1993; Blagoveshchensky, 2011]. This information about signal propagation in a real randomly inhomogeneous ionosphere is known with a certain degree of probability. On the other hand, fluctuations of characteristics of a probe signal, which passed through the ionospheric channel, carry information about random irregularities of the medium. Therefore, the reliable use of the ionospheric channel needs diagnostics of expected characteristics of the main signal directed to a destination site, from measurements of characteristics of the probe counter signal received by the transmitter of the main signal. One solution to these issues is the approach based on direct diagnostics of the ionospheric radio channel, when parameters of a probe signal are recalculated into the main characteristics of the main transmitted signal [Kurkin et al., 1993]. It provides important results concerning prediction of maximum usable frequencies and distance-frequency characteristics of a decameter signal under different geophysical conditions. The accuracy of the real-time prediction, which is based on the direct diagnostics of the radio channel, largely depends on strictness of the functional relations that connect characteristics of probe and transmitted signals. In general, such relations are obtained for a regular medium regardless of its fine chaotic structure, therefore it is interest- ing to derive such relations in the problem of signal propagation in a randomly inhomogeneous ionosphere.

In the general case, the decameter ionospheric radio channel is a complex multiply connected system [Mitra, 1947; Davies, 1990]. Due to significant anisotropy of the ionosphere, multiscale nature of irregularities, and features of radio wave propagation mechanisms, the diagnostics of the ionospheric channel is a great problem and is highly relevant [Kazantsev et al., 1967; Blagoveshchensky, Zherebtsov, 1987; Kurkin et al., 1993; Alimov et al., 1997; Kryukovskii et al., 2012, Kryukovsky et al., 2016; Ipatov et al., 2014; Bova et al., 2019]. In particular, when assessing statistical characteristics of a decameter signal, a question arises about the shape of the spectrum of the channel's random irregularities. There is no clarity about the spatial distribution of the irregularities over the entire ionosphere. Meanwhile, the problem of estimating statistical characteristics of the decameter signal in the ionospheric radio channel can be solved using a model of randomly inhomogeneous ionosphere with generalized (integral) features. In this regard, significant results have been obtained due to the introduction of concepts about the effective correlation ellipsoid that approximately describes random irregularities of the medium [Gusev, Ovchinnikova, 1980; Vologdin et al., 2007, 2012; Afanasiev et al., 2009] and significantly simplifies analytical calculations of statistical moments of a signal. Although the ionosphere is a multiscale randomly inhomogeneous medium and features a power spectrum of ir- 
regularities, in some cases, when calculating the lowest moments of trajectory characteristics of a signal, we can use a Gaussian correlation ellipsoid of irregularities with effective parameters. In particular, the studies [Alimov et al., 1997; Afanasiev et al., 2009; Afanasiev et al., 2010] have shown that when calculating the phase variance of a decameter radio signal in a multiscale randomly inhomogeneous medium we can adopt a Gaussian correlation ellipsoid model provided that as the spatial scale of irregularities we take the external scale of the ionospheric turbulence given by the power spectrum. This is due to the fact that the high-frequency part of the spectrum of irregularities affects to a greater extent the signal amplitude and to a lesser extent its phase [Ishimaru, 1999].

Ageeva et al. [2020] have explored the possibility of estimating the statistical characteristics of a signal in a two-dimensional channel from measurements of trajectory moments of a probe signal. In this paper, we develop this approach for a three-dimensional case and use it to calculate the expected statistical characteristics of decameter signals along single-hop ionospheric paths.

\section{BASIC THEORETICAL RELATIONS}

Suppose that at a point $\mathrm{I}_{0}$ located on Earth's surface (Figure 1) is a source of a quasi-monochromatic decameter signal. Estimate the expected statistical trajectory characteristics of the signal on a given ionospheric path $\mathrm{I}_{0} \mathrm{P}_{0}$ (path length $x_{\mathrm{k}}$ ). For this purpose, from a point $\mathrm{I}_{\mathrm{v}}$, located in the vicinity of $\mathrm{P}_{0}$, send a probe signal to the source of the main signal. Assume that trajectory characteristics of the probe signal can be measured at a point $\mathrm{P}_{\mathrm{v}}$ coinciding with $\mathrm{I}_{0}$. Obtain approximate analytical relations relating statistical trajectory characteristics of signals on the main path $\mathrm{I}_{0} \mathrm{P}_{0}$ and on a secondary path $\mathrm{I}_{\mathrm{v}}$ $\mathrm{P}_{\mathrm{v}}$ (path length $x_{\mathrm{p}}$ ). Consider the case of signal propagation in a randomly inhomogeneous ionosphere without regard to the geomagnetic field and Earth curvature. We need these simplifications to more clearly and explicitly demonstrate the potential of the proposed approximate method for estimating the expected statistical characteristics of a signal along a single-hop path of average length at the first stage of solving the given general problem.

As measured characteristics of the probe decameter signal transmitted from $I_{v}$ and received by the transmitter of the main signal $\mathrm{I}_{0}$ we will use second-order statistical moments of phase, group delay, and Doppler frequency shift. To restore parameters of the correlation ellipsoid of ionospheric irregularities from measurements

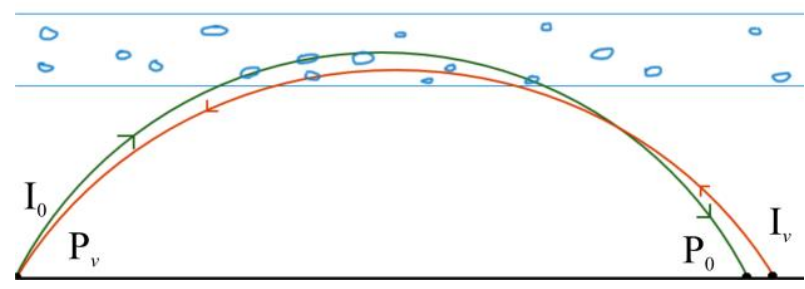

Figure 1. Scheme of diagnostics of the ionospheric path $\mathrm{I}_{0} \mathrm{P}_{0}$ from measurements along the $\mathrm{I}_{\mathrm{v}} \mathrm{P}_{\mathrm{v}}$ path of characteristics of the probe signal on the secondary path $\mathrm{I}_{\mathrm{v}} \mathrm{P}_{\mathrm{v}}$, derive the functional relations that relate them with due regard to the boundary conditions at reception and transmission points.

In the ray approximation [Kravtsov, Orlov, 1980] for fluctuations of phase, group delay, and Doppler frequency shift of a signal, which propagates in an isotropic three-dimensional inhomogeneous ionosphere, in the Cartesian coordinate system we have [Gershman et al., 1984]

$$
\begin{aligned}
& \varphi=\frac{2 \pi f}{c} \int_{0}^{x_{\mathrm{p}}} \sqrt{\varepsilon} \sqrt{1+\left(\frac{d y}{d x}\right)^{2}+\left(\frac{d z}{d x}\right)^{2}} d x, \\
& t=\int_{0}^{x_{\mathrm{p}}} \frac{1}{c \sqrt{\varepsilon}} \sqrt{1+\left(\frac{d y}{d x}\right)^{2}+\left(\frac{d z}{d x}\right)^{2}} d x, \\
& \Delta f=-\frac{f}{c} \frac{\partial}{\partial \tau} \int_{0}^{x_{\mathrm{p}}} \sqrt{\varepsilon} \sqrt{1+\left(\frac{d y}{d x}\right)^{2}+\left(\frac{d z}{d x}\right)^{2}} d x,
\end{aligned}
$$

where $\varepsilon=\varepsilon(x, y, z, \tau)$ is the spatiotemporal random function of permittivity; $\tau$ is the time; $f$ is the operating frequency; $c$ is the velocity of light; $x_{\mathrm{p}}$ is the length of the secondary path, and integration is performed along random ray trajectories, which connect points of transmission and reception of the probe signal. Different methods of constructing ray trajectories are well known [Kazantsev et al., 1967; Kravtsov, Orlov, 1980; Klyatskin, 2008; Kryukovskii et al., 2012]. To calculate the rays, we use the Euler system of differential equations with independent variable of range element $d x$ [Tereschenko, 1971]

$$
\left\{\begin{array}{l}
\frac{d z}{d x}=\operatorname{ctg} \beta \\
\frac{d \beta}{d x}=\frac{1}{\sqrt{\varepsilon}}\left(1+\sin ^{2} \beta \operatorname{tg}^{2} \alpha\right)\left(\frac{\partial \sqrt{\varepsilon}}{\partial x} \operatorname{ctg} \beta-\frac{\partial \sqrt{\varepsilon}}{\partial z}\right) \\
\frac{d \alpha}{d x}=\frac{1}{\sqrt{\varepsilon}}\left(1+\cos ^{2} \alpha \operatorname{ctg}^{2} \beta\right)\left(\frac{\partial \sqrt{\varepsilon}}{\partial y}-\frac{\partial \sqrt{\varepsilon}}{\partial x} \operatorname{tg} \alpha\right),
\end{array}\right.
$$

where $x, y, z$ are current ray coordinates; $\alpha, \beta$ are refraction angles in the azimuth and elevation planes. Compared to ray equations in Hamiltonian form [Ageeva et al., 2020], where the independent variable is a group delay element, the Euler form can more compactly and graphically represent the results of calculations of characteristics of oblique radio wave propagation in the randomly inhomogeneous ionosphere.

Find statistical moments of the trajectory characteristics of the probe signal on the secondary path $\mathrm{I}_{\mathrm{v}} \mathrm{P}_{\mathrm{v}}$ in the approximation of the perturbation method [Arnold, 1989]. For functions in Equations (1)-(4), use the expansions

$$
\left\{\begin{array}{l}
\varepsilon=\varepsilon_{0}(z)+\varepsilon_{1}(x, y, z, \tau) \\
\varphi=\varphi_{0}+\varphi_{1}, t=t_{0}+t_{1} \\
\Delta f=\Delta f_{0}+\Delta f_{1} \\
z=z_{0}+z_{1}, y=y_{0}+y_{1} \\
\beta=\beta_{0}+\beta_{1}, \alpha=\alpha_{0}+\alpha_{1},
\end{array}\right.
$$


where $y_{0}, z_{0}, \alpha_{0}, \beta_{0}, \varphi_{0}, t_{0}, \Delta f_{0}, y_{1}, z_{1}, \alpha_{1}, \beta_{1}, \varphi_{1}, t_{1}, \Delta f_{1}$ are signal mean and fluctuation characteristics; $\varepsilon_{0}$ describes mean permittivity of the radio channel; $\varepsilon_{1}$ characterizes spatiotemporal random ionospheric irregularities.

From HF communication it is known that under quiet geophysical conditions during oblique ionospheric sounding radio waves usually propagate in the plane of the great-circle arc [Rawer, 1993]. Assume, therefore, that the wave field is more regular in the transverse plane than in the vertical one. For simplicity, consider the mean ray trajectory lying in the plane of the greatcircle arc (in our case, in the XOZ plane $\left(\alpha_{0}=0, y_{0}=0\right)$ ). Substituting (5) into (1)-(4) and performing calculations with due regard to boundary conditions at reception and transmission points, we derive generating equations

$$
\begin{aligned}
& \varphi_{0}=\frac{2 \pi f}{c} \int_{0}^{x_{\mathrm{p}}} \sqrt{\varepsilon_{0}} \sqrt{1+\left(\frac{d z_{0}}{d x}\right)^{2}} d x, \\
& t_{0}=\int_{0}^{x_{\mathrm{p}}} \frac{1}{c \sqrt{\varepsilon_{0}}} \sqrt{1+\left(\frac{d z_{0}}{d x}\right)^{2}} d x, \\
& \Delta f_{0}=-\frac{f}{c} \frac{\partial}{\partial \tau} \int_{0}^{x_{\mathrm{p}}} \sqrt{\varepsilon_{0}} \sqrt{1+\left(\frac{d z_{0}}{d x}\right)^{2}} d x=0
\end{aligned}
$$

and fluctuation equations

$$
\begin{aligned}
\varphi_{1} & =\frac{\pi f}{c} \int_{0}^{x_{\mathrm{p}}} \frac{\varepsilon_{1}}{\sqrt{\varepsilon_{0}}} \frac{d x}{\sin \beta_{0}} \\
t_{1} & =\int_{0}^{x_{\mathrm{p}}} \frac{F_{\mathrm{p}}(x)}{c \sqrt{\varepsilon_{0}} \sin \beta_{0}} \frac{\partial \varepsilon_{1}}{\partial z_{0}} d x-\frac{1}{2} \int_{0}^{x_{\mathrm{p}}} \frac{\varepsilon_{1}}{c \varepsilon_{0} \sqrt{\varepsilon_{0}} \sin \beta_{0}} d x, \\
\Delta f_{1} & =-\frac{f}{2 c} \int_{0}^{x_{\mathrm{p}}} \frac{1}{\sqrt{\varepsilon_{0}} \sin \beta_{0}} \frac{\partial \varepsilon_{1}}{\partial \tau} d x
\end{aligned}
$$

where

$$
\begin{aligned}
& F_{\mathrm{p}}(x)=F_{1 \mathrm{p}}(x)+F_{2 \mathrm{p}}(x) \\
& F_{1 \mathrm{p}}(x)=\frac{c}{2 \sin \beta_{\mathrm{p}} R_{\mathrm{lp}}\left(x_{\mathrm{p}}\right)} R_{2 \mathrm{p}}(x) P_{1 \mathrm{p}}(x) \\
& F_{2 \mathrm{p}}(x)=\frac{c}{2 \sin \beta_{\mathrm{p}} R_{\mathrm{lp}}\left(x_{\mathrm{p}}\right)} R_{\mathrm{lp}}(x) P_{2 \mathrm{p}}(x) \\
& P_{1 \mathrm{p}}(x)=\int_{0}^{x} \frac{\sin \beta_{0}}{\varepsilon_{0}} \frac{\partial \varepsilon_{0}}{\partial z_{0}} R_{1 \mathrm{p}}(x) \frac{d x}{c \sqrt{\varepsilon_{0}}} \\
& P_{2 \mathrm{p}}(x)=\int_{x}^{x_{\mathrm{p}}} \frac{\sin \beta_{0}}{\varepsilon_{0}} \frac{\partial \varepsilon_{0}}{\partial z_{0}} R_{2 \mathrm{p}}(x) \frac{d x}{c \sqrt{\varepsilon_{0}}} \\
& R_{1 \mathrm{p}}=\frac{\partial z_{0}}{\partial \beta_{\mathrm{p}}}(x), \quad R_{2 \mathrm{p}}=\frac{\partial z_{0}}{\partial \beta_{\mathrm{p}}}\left(x_{\mathrm{p}}-x\right) \text { are fundamental }
\end{aligned}
$$

solutions of the boundary-value trajectory problem for a probe source; $\beta_{\mathrm{p}}$ is the angle of entry of the probe signal into the channel (measured from vertical), the $\mathrm{p}$ index characterizes the probe path. Integration in (6)-(11), (15), (16) is performed over the mean trajectory, which is the solution of the system of equations

$$
\left\{\frac{d z_{0}}{d x}=\operatorname{ctg} \beta_{0}, \frac{d \beta_{0}}{d x}=-\frac{1}{\sqrt{\varepsilon_{0}}} \frac{\partial \sqrt{\varepsilon_{0}}}{\partial z_{0}}\right.
$$

with initial conditions $z_{0}\left(x_{\mathrm{p}}\right)=0, \beta_{0}\left(x_{\mathrm{p}}\right)=\beta_{\mathrm{p}}$.

From (9)-(11) find the statistical moments of the trajectory characteristics of the probe signal on the secondary path $x_{\mathrm{p}}$ long. Consider conditions of a quasihomogeneous random field of the channel's irregularities. Set the correlation function of permittivity fluctuations to be [Gershman et al., 1984]

$$
N=\left\langle\varepsilon_{1}\left(x_{1}, y_{1}, z_{1}, \tau_{1}\right) \varepsilon_{1}\left(x_{2}, y_{2}, z_{2}, \tau_{2}\right)\right\rangle=N_{1} N_{0},
$$

where $N_{0}$ is a homogeneous part of the correlation function. The function $N_{1}$ represents a statistical irregularity of the random field of irregularities and takes into account variability of irregularity parameters in the channel, with $N_{1}$ varying more slowly than $N_{0}$. As the $N_{1}$ function consider the relationship $N_{1}=\mu^{2}\left(1-\varepsilon_{0}\right)^{2}$, where $\mu^{2}$ is the intensity of random irregularities of the ionospheric electron density. Take into account the motion of irregularities in terms of the hypothesis of frozen transfer:

$$
\begin{aligned}
& N_{0}=\exp \left(-\frac{1}{a^{2}}\left[\left(x_{1}-x_{2}\right)^{2}+\left(y_{1}-y_{2}\right)^{2}+\right.\right. \\
& \left.\left.+\left(z_{1}-z_{2}-V\left(\tau_{1}-\tau_{2}\right)\right)^{2}\right]\right),
\end{aligned}
$$

where $a$ is the scale of irregularities; $V$ is the velocity of motion of the random field of irregularities. By finding statistical moments from (9)-(11) and carrying out analytical transformations, for the selected model of correlation function of irregularities we obtain integral expressions for variances of signal trajectory characteristics

$$
\begin{aligned}
& \sigma_{\varphi \mathrm{p}}^{2}=\int_{0}^{x_{\mathrm{p}}} \frac{\sqrt{\pi} \omega^{2} a \mu^{2}\left(1-\varepsilon_{0}\right)^{2}}{4 c^{2} \sqrt{\varepsilon_{0}} \sin \beta_{\mathrm{p}}} d x, \\
& \sigma_{f \mathrm{p}}^{2}=\int_{0}^{x_{\mathrm{p}}} \frac{f^{2} \sqrt{\pi} V^{2} \mu^{2}\left(1-\varepsilon_{0}\right)^{2}}{2 a} \frac{\sin \beta_{0}}{c^{2} \varepsilon_{0}} d x, \\
& \sigma_{\Delta t \mathrm{p}}^{2}=2 \int_{0}^{x_{\mathrm{p}}}\left[F_{\mathrm{p}}(x)\right]^{2} \frac{\mu^{2}\left(1-\varepsilon_{0}\right)^{2}}{\varepsilon_{0}^{2} \sin \beta_{0}} \sin ^{2} \beta_{\mathrm{p}} \frac{\sqrt{\pi}}{a c^{2}} d x+ \\
& +\int_{0}^{x_{\mathrm{p}}} \frac{\sqrt{\pi} a}{4 c^{2}} \frac{\mu^{2}\left(1-\varepsilon_{0}\right)^{2}}{\varepsilon_{0}^{3} \sin \beta_{0}} d x,
\end{aligned}
$$

where $\omega=2 \pi f$. Solving Equations (20)-(22) relative to unknown parameters of the correlation ellipsoid, we get

$$
\begin{aligned}
& \mu^{2}=\sqrt{\frac{J_{1} \sigma_{\Delta t \mathrm{p}}^{2}-J_{3} \sigma_{\varphi \mathrm{p}}^{2}}{J_{2} J_{1}^{2}}}, \\
& a=\frac{\sqrt{J_{2} \sigma_{\varphi p}^{2}}}{\sqrt{J_{1} \sigma_{\Delta t \mathrm{p}}^{2}-J_{3} \sigma_{\varphi p}^{2}}}, \\
& V=\sqrt{\frac{\sigma_{f \mathrm{p}}^{2} J_{1} J_{2}}{J_{4}\left(J_{1} \sigma_{\Delta t \mathrm{p}}^{2}-J_{3} \sigma_{\varphi \mathrm{p}}^{2}\right)}},
\end{aligned}
$$

where 


$$
\begin{aligned}
& J_{1}=\frac{\sqrt{\pi} \omega^{2}}{4 c^{2} \sin \beta_{\mathrm{p}}} \int_{0}^{x_{\mathrm{p}}} \frac{\left(1-\varepsilon_{0}\right)^{2} d x}{\sqrt{\varepsilon_{0}}}, \\
& J_{2}=\frac{2 \sin \beta_{\mathrm{p}} \sqrt{\pi}}{c^{2}} \int_{0}^{x_{\mathrm{p}}} \frac{\left(1-\varepsilon_{0}\right)^{2} F_{\mathrm{p}}^{2} d x}{\sqrt{\varepsilon_{0}^{3}}}, \\
& J_{3}=\frac{\sqrt{\pi}}{4 c^{2} \sin \beta_{\mathrm{p}}} \int_{0}^{x_{\mathrm{p}}} \frac{\left(1-\varepsilon_{0}\right)^{2} d x}{\sqrt{\varepsilon_{0}^{5}}}, \\
& J_{4}=\frac{f^{2} \sqrt{\pi}}{2 c^{2} \sin \beta_{\mathrm{p}}} \int_{0}^{x_{\mathrm{p}}} \frac{\sin ^{2} \beta_{0}\left(1-\varepsilon_{0}\right)^{2} d x}{\sqrt{\varepsilon_{0}}} .
\end{aligned}
$$

It is noteworthy that the integrals $J_{1}, J_{2}, J_{3}, J_{4}$ are independent of parameters of the correlation ellipsoid of irregularities and are formed only by mean trajectory characteristics of a probe signal on the path $\mathrm{I}_{\mathrm{v}} \mathrm{P}_{\mathrm{v}}$, which depend on properties of permittivity of the averaged ionosphere $\varepsilon_{0}$.

After determining the ellipsoid parameters on the secondary path $\mathrm{I}_{\mathrm{v}} \mathrm{P}_{\mathrm{v}}$, we can compute the expected statistical characteristics of the main signal at the given path $\mathrm{I}_{0} \mathrm{P}_{0}$. Using the same mathematical apparatus as above, for the moments of the trajectory characteristics of the main signal on the path $\mathrm{I}_{0} \mathrm{P}_{0}$, obtain

$$
\begin{aligned}
& \sigma_{\varphi}^{2}=\int_{0}^{x_{\mathrm{k}}} \frac{\sqrt{\pi} \omega^{2} a \mu^{2}\left(1-\varepsilon_{0}\right)^{2}}{4 c^{2} \sqrt{\varepsilon_{0}} \sin \beta_{\mathrm{n}}} d x, \\
& \sigma_{f}^{2}=\int_{0}^{x_{\mathrm{k}}} \frac{f^{2} \sqrt{\pi} V^{2} \mu^{2}\left(1-\varepsilon_{0}\right)^{2}}{2 a} \frac{\sin \beta_{0}}{c^{2} \varepsilon_{0}} d x, \\
& \sigma_{\Delta t}^{2}=2 \int_{0}^{x_{\mathrm{k}}}[F(x)]^{2} \frac{\mu^{2}\left(1-\varepsilon_{0}\right)^{2}}{\varepsilon_{0}^{2} \sin \beta_{0}} \sin ^{2} \beta_{\mathrm{n}} \frac{\sqrt{\pi}}{a c^{2}} d x+ \\
&+\int_{0}^{x_{\mathrm{k}}} \frac{\sqrt{\pi} a}{4 c^{2}} \frac{\mu^{2}\left(1-\varepsilon_{0}\right)^{2}}{\varepsilon_{0}^{3} \sin \beta_{0}} d x,
\end{aligned}
$$

where

$$
\begin{aligned}
& F(x)=F_{1}(x)+F_{2}(x) \\
& F_{1}(x)=\frac{c}{2 \sin \beta_{\mathrm{n}} R_{1}\left(x_{\kappa}\right)} R_{2}(x) P_{1}(x), \\
& F_{2}(x)=\frac{c}{2 \sin \beta_{\mathrm{n}} R_{1}\left(x_{\kappa}\right)} R_{1}(x) P_{2}(x) \\
& P_{1}(x)=\int_{0}^{x} \frac{\sin \beta_{0}}{\varepsilon_{0}} \frac{\partial \varepsilon_{0}}{\partial z_{0}} \frac{R_{1}(x)}{c \sqrt{\varepsilon_{0}}} d x \\
& P_{2}(x)=\int_{x}^{x_{\mathrm{\kappa}}} \frac{\sin \beta_{0}}{\varepsilon_{0}} \frac{\partial \varepsilon_{0}}{\partial z_{0}} \frac{R_{2}(x)}{c \sqrt{\varepsilon_{0}}} d x . \\
& R_{1}=\frac{\partial z_{0}}{\partial \beta_{\mathrm{n}}}(x), R_{2}=\frac{\partial z_{0}}{\partial \beta_{\mathrm{n}}}\left(x_{\mathrm{\kappa}}-x\right) \text { are fundamental solu- }
\end{aligned}
$$

tions of the boundary-value trajectory problem for the main source, $\beta_{\mathrm{n}}$ is the angle of entry of the main signal into the channel, the index $\mathrm{n}$ describes the given path. Integration in (30)-(32), (36), (37) is performed over the mean trajectory, which is the solution of system of equations (17), with initial conditions $z_{0}(0)=0, \beta_{0}(0)=\beta_{\mathrm{n}}$.

By substituting correlation ellipsoid parameters (23)-(25) into (30)-(32) and making analytic transformation, have:

$$
\begin{aligned}
& \sigma_{\varphi}^{2}=\frac{G_{1}}{J_{1}} \sigma_{\varphi p}^{2}, \\
& \sigma_{f}^{2}=\frac{G_{4}}{J_{4}} \sigma_{f \mathrm{p}}^{2}, \\
& \sigma_{\Delta t}^{2}=\frac{G_{2}}{J_{2}} \sigma_{\Delta t \mathrm{p}}^{2}+\sigma_{\varphi \mathrm{p}}^{2}\left(\frac{G_{3}}{J_{1}}-\frac{G_{2} J_{3}}{J_{2} J_{1}}\right),
\end{aligned}
$$

where

$$
\begin{aligned}
& G_{1}=\frac{\sqrt{\pi} \omega^{2}}{4 c^{2} \sin \beta_{\mathrm{n}}} \int_{0}^{x_{\kappa}} \frac{\left(1-\varepsilon_{0}\right)^{2} d x}{\sqrt{\varepsilon_{0}}}, \\
& G_{2}=\frac{2 \sin \beta_{\mathrm{n}} \sqrt{\pi}}{c^{2}} \int_{0}^{x_{\kappa}} \frac{\left(1-\varepsilon_{0}\right)^{2} F^{2} d x}{\sqrt{\varepsilon_{0}^{3}}}, \\
& G_{3}=\frac{\sqrt{\pi}}{4 c^{2} \sin \beta_{\mathrm{n}}} \int_{0}^{x_{\kappa}} \frac{\left(1-\varepsilon_{0}\right)^{2} d x}{\sqrt{\varepsilon_{0}^{5}}}, \\
& G_{4}=\frac{f^{2} \sqrt{\pi}}{2 c^{2} \sin \beta_{\mathrm{n}}} \int_{0}^{x_{\kappa}} \frac{\sin ^{2} \beta_{0}\left(1-\varepsilon_{0}\right)^{2} d x}{\sqrt{\varepsilon_{0}}} .
\end{aligned}
$$

Here, the integrals $G_{1}, G_{2}, G_{3}, G_{4}$ depend only on properties of permittivity $\varepsilon_{0}$ and are formed by trajectory characteristics of the main signal on the path $\mathrm{I}_{0} \mathrm{P}_{0}$ in the averaged ionosphere.

Relations (38)-(40) represent an explicit link between statistical characteristics of the main and probe signals along oblique ionospheric sounding paths. By measuring $\sigma_{\varphi p}^{2}, \sigma_{f p}^{2}, \sigma_{\Delta t p}^{2}$ of the probe signal on the secondary path $\mathrm{I}_{\mathrm{v}} \mathrm{P}_{\mathrm{v}}$ and setting the mean vertical profile of permittivity $\varepsilon_{0}$ of the ionosphere, we can use Formulas (38)-(40) to calculate the expected statistical trajectory characteristics of the signal on the given path $\mathrm{P}_{0} \mathrm{I}_{0}$.

\section{ALGORITHMIZATION OF DESIGN FORMULAS}

To quantitatively estimate $\sigma_{\varphi}^{2}, \sigma_{f}^{2}, \sigma_{\Delta t}^{2}$ from (38)(40), it is necessary to calculate integral coefficients (26)-(29), (41)-(44). The direct numerical calculation is rather complex because it requires us to know all integrands including fundamental solutions along mean trajectories on the main and secondary paths. Meanwhile, by setting upper limits of integrals (26)-(29), (41)-(44) to be variable and by differentiating the integrals over these limits, we derive differential equations that can be numerically integrated along with (17). Through these transformations, to calculate (26)-(29), (41)-(44) we have the following differential equations:

$$
\frac{d J_{1}}{d x}=\frac{\sqrt{\pi} \omega^{2}}{4 c^{2} \sin \beta_{\mathrm{p}}} \frac{\left(1-\varepsilon_{0}\right)^{2}}{\sqrt{\varepsilon_{0}}},
$$




$$
\begin{aligned}
& \frac{d J_{2}}{d x}=\frac{2 \sin \beta_{\mathrm{p}} \sqrt{\pi}}{c^{2}} \frac{\left(1-\varepsilon_{0}\right)^{2} F_{\mathrm{p}}^{2}}{\sqrt{\varepsilon_{0}^{3}}}, \\
& \frac{d J_{3}}{d x}=\frac{\sqrt{\pi}}{4 c^{2} \sin \beta_{\mathrm{p}}} \frac{\left(1-\varepsilon_{0}\right)^{2}}{\sqrt{\varepsilon_{0}^{5}}}, \\
& \frac{d J_{4}}{d x}=\frac{f^{2} \sqrt{\pi}}{2 c^{2} \sin \beta_{\mathrm{p}}} \frac{\sin ^{2} \beta_{0}\left(1-\varepsilon_{0}\right)^{2}}{\sqrt{\varepsilon_{0}}}, \\
& \frac{d G_{1}}{d x}=\frac{\sqrt{\pi} \omega^{2}}{4 c^{2} \sin \beta_{\mathrm{n}}} \frac{\left(1-\varepsilon_{0}\right)^{2}}{\sqrt{\varepsilon_{0}}}, \\
& \frac{d G_{2}}{d x}=\frac{2 \sin \beta_{\mathrm{n}} \sqrt{\pi}}{c^{2}} \frac{\left(1-\varepsilon_{0}\right)^{2} F^{2}}{\sqrt{\varepsilon_{0}^{3}}}, \\
& \frac{d G_{3}}{d x}=\frac{\sqrt{\pi}}{4 c^{2} \sin \beta_{\mathrm{n}}} \frac{\left(1-\varepsilon_{0}\right)^{2}}{\sqrt{\varepsilon_{0}^{5}}}, \\
& \frac{d G_{4}}{d x}=\frac{f^{2} \sqrt{\pi}}{2 c^{2} \sin \beta_{\mathrm{n}}} \frac{\sin ^{2} \beta_{0}\left(1-\varepsilon_{0}\right)^{2}}{\sqrt{\varepsilon_{0}}}
\end{aligned}
$$

with initial conditions:

$$
\begin{aligned}
& J_{1}\left(x_{\mathrm{p}}\right)=0, \quad J_{2}\left(x_{\mathrm{p}}\right)=0, \quad J_{3}\left(x_{\mathrm{p}}\right)=0, \quad J_{4}\left(x_{\mathrm{p}}\right)=0, \\
& G_{1}(0)=0, G_{2}(0)=0, G_{3}(0)=0, G_{4}(0)=0 .
\end{aligned}
$$

Equations (46), (50) include functions $F_{\mathrm{p}}(x), F(x)$, which are defined by Formulas (12), (33). In turn, (12), (33) include integrals (15), (16), (36), (37), which we can also reduce to differential equations by differentiating with respect to the variable limit $x$. Given Snell's law [Kravtsov, Orlov, 1980], we have

$$
\begin{aligned}
& \frac{d P_{1 \mathrm{p}}}{d x}=\frac{\sin \beta_{\mathrm{p}}}{\varepsilon_{0}^{2}} \frac{\partial \varepsilon_{0}}{\partial z} \frac{R_{\mathrm{lp}}(x)}{c}, \\
& \frac{d P_{2 \mathrm{p}}}{d x}=-\frac{\sin \beta_{\mathrm{p}}}{\varepsilon_{0}^{2}} \frac{\partial \varepsilon_{0}}{\partial z} \frac{R_{2 \mathrm{p}}(x)}{c}, \\
& \frac{d P_{1}}{d x}=\frac{\sin \beta_{\mathrm{n}}}{\varepsilon_{0}^{2}} \frac{\partial \varepsilon_{0}}{\partial z} \frac{R_{1}(x)}{c}, \\
& \frac{d P_{2}}{d x}=-\frac{\sin \beta_{\mathrm{n}}}{\varepsilon_{0}^{2}} \frac{\partial \varepsilon_{0}}{\partial z} \frac{R_{2}(x)}{c} .
\end{aligned}
$$

Proper initial conditions for (53)-(56) have the form $P_{1 \mathrm{p}}\left(x_{\mathrm{p}}\right)=0, P_{2 \mathrm{p}}(0)=0, P_{1}(0)=0, P_{2}\left(x_{\mathrm{k}}\right)=0$. Finally, the fundamental solutions $R_{1}(x), R_{2}(x), R_{1 \mathrm{p}}(x), R_{2 \mathrm{p}}(x)$ in (13)(16), (34)-(37) can be determined by integrating the system of equations, derived by alternately differentiating ray equations (17) with respect to the parameters $\beta_{\mathrm{n}}$ and $\beta_{\mathrm{p}}$,

$$
\left\{\begin{array}{l}
\frac{d R_{1}}{d x}=-\frac{\varepsilon_{0} Q_{1}}{\sin ^{2} \beta_{\mathrm{n}}}, \frac{d Q_{1}}{d x}=W R_{1} \\
\frac{d R_{2}}{d x}=-\frac{\varepsilon_{0} Q_{2}}{\sin ^{2} \beta_{\mathrm{n}}}, \frac{d Q_{2}}{d x}=W R_{2}
\end{array}\right.
$$

$$
\left\{\begin{array}{l}
\frac{d R_{1 \mathrm{p}}}{d x}=-\frac{\varepsilon_{0} Q_{1 \mathrm{p}}}{\sin ^{2} \beta_{\mathrm{p}}}, \frac{d Q_{1 \mathrm{p}}}{d x}=W R_{\mathrm{lp}} \\
\frac{d R_{2 \mathrm{p}}}{d x}=-\frac{\varepsilon_{0} Q_{2 \mathrm{p}}}{\sin ^{2} \beta_{\mathrm{p}}}, \frac{d Q_{2 \mathrm{p}}}{d x}=W R_{2 \mathrm{p}}
\end{array}\right.
$$

where $\quad W=-\frac{\partial}{\partial z_{0}}\left(\frac{1}{\sqrt{\varepsilon_{0}}}\left(\frac{\partial \sqrt{\varepsilon_{0}}}{\partial z_{0}}\right)\right), \quad Q_{1}=\frac{\partial \beta_{0}}{\partial \beta_{\mathrm{n}}}(x)$,

$Q_{2}=\frac{\partial \beta_{0}}{\partial \beta_{\mathrm{n}}}\left(x_{\kappa}-x\right), Q_{\mathrm{p}}=\frac{\partial \beta_{0}}{\partial \beta_{\mathrm{p}}}(x), Q_{2 \mathrm{p}}=\frac{\partial \beta_{0}}{\partial \beta_{\mathrm{p}}}\left(x_{\mathrm{p}}-x\right)$.

The initial conditions for (57), (58) have the form $R_{1}(0)=0, \quad Q_{1}(0)=1, \quad R_{2} \quad\left(x_{\mathrm{k}}\right)=0, \quad Q_{2} \quad\left(x_{\mathrm{k}}\right)=1, \quad R_{1 \mathrm{p}}\left(x_{\mathrm{p}}\right)=0$, $Q_{1 \mathrm{p}}\left(x_{\mathrm{p}}\right)=1, R_{2 \mathrm{p}}(0)=0, Q_{2 \mathrm{p}}(0)=1$.

By grouping Equations (17), (45)-(58) for the main and probe sources, we have two independent systems of differential equations to calculate integral coefficients along the given and secondary paths.

\section{RESULTS OF NUMERICAL EXPERIMENTS}

The method for direct diagnostics of the stochastic ionospheric channel has been tested in numerical experiments. To estimate the expected variances of trajectory characteristics along given single-hop paths, as input data we have used results of calculations of probe signal trajectory moments on the secondary path, obtained with known parameters of ionospheric irregularities. A key question concerning the implementation of the proposed method is the correct choice of secondary path to compute signal characteristics on the main path. As mentioned above, a transmitter of a probe signal should be at a distance from a receiver of the main signal, and a receiver of an opposite probe signal should coincide with a transmitter of the main signal. Rough ideas about possible probe paths to assess statistical characteristics of a signal on the main path in the typical ionosphere can be obtained by modeling the regular trajectory pattern on the basis of Equations (17). The analysis of these calculations has shown that depending on the type of the mean profile of ionospheric permittivity for a given inclined path we can found a number of such paths. As an example Figure 2 presents decameter signal paths in the two-layer ionosphere specified by an analytical model

$$
\begin{aligned}
& \varepsilon_{0}\left(z_{0}\right)=1-\frac{f_{\mathrm{kp} E}^{2}}{f^{2}} \exp \left(-\left(\frac{z_{0}-z_{\mathrm{mE}}}{y_{\mathrm{mE}}}\right)^{2}\right)- \\
& -\frac{f_{\mathrm{kp}}^{2}}{f^{2}} \exp \left(-\left(\frac{z_{0}-z_{\mathrm{m}}}{y_{\mathrm{m}}}\right)^{2}\right)
\end{aligned}
$$

where $\mathrm{z}_{\mathrm{mE}}, \mathrm{z}_{\mathrm{m}}, \mathrm{y}_{\mathrm{mE}}, \mathrm{y}_{\mathrm{m}}, \mathrm{f}_{\mathrm{crE}}, \mathrm{f}_{\mathrm{cr}}$ are heights of ionization maxima, half-thickness, and critical frequencies of $\mathrm{E}$ and F2 layers respectively. Parameters of the model: $z_{\mathrm{mE}}=150 \mathrm{~km}, z_{\mathrm{m}}=320 \mathrm{~km}, y_{\mathrm{mE}}=35 \mathrm{~km}, y_{\mathrm{m}}=120 \mathrm{~km}, f_{\mathrm{crE}}=$ $=4 \mathrm{MHz}, f_{\mathrm{cr}}=8 \mathrm{MHz}$. The operating frequency $f=15 \mathrm{MHz}$. Trajectories are calculated in the sector of $60^{\circ}-80^{\circ}$ angles 


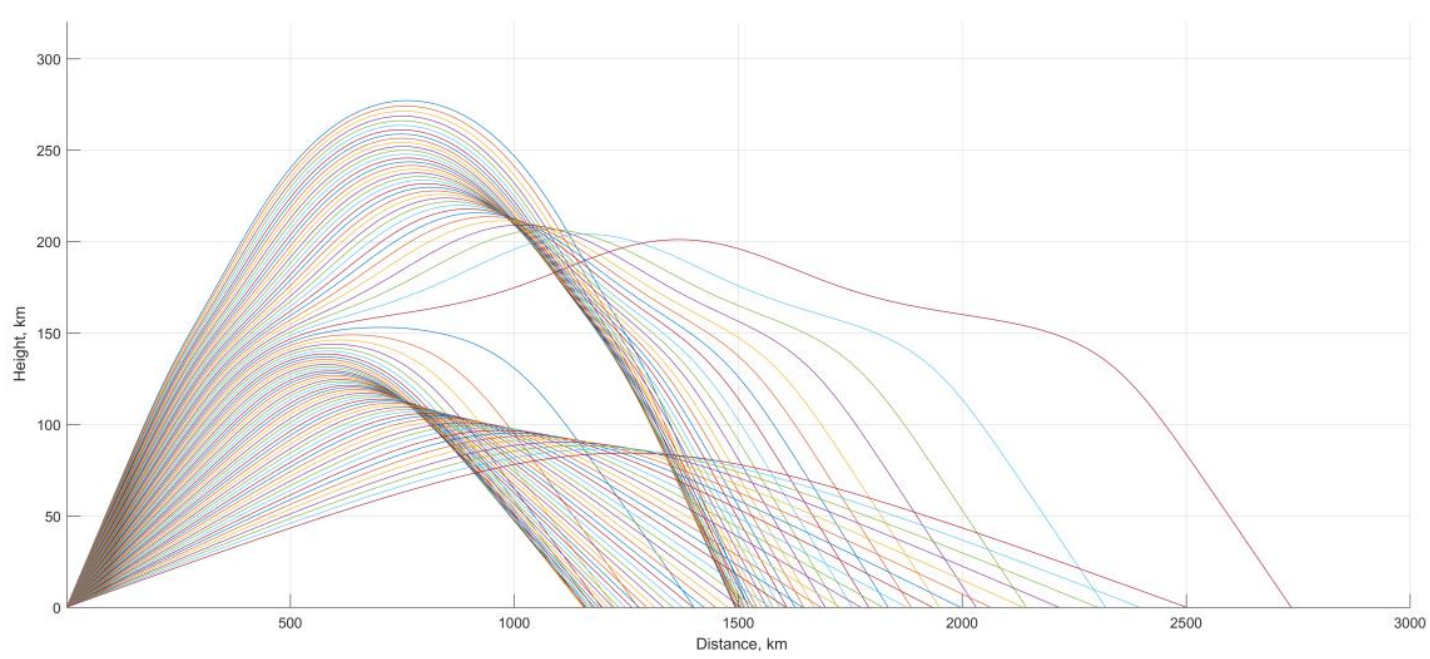

Figure 2. Typical ray pattern in the two-layer ionosphere

of entry into the channel with a step of $0.3^{\circ}$. Figure 2 shows that, for example, for the main path $\mathrm{x}_{c r}=1600 \mathrm{~km}$ (F2 mode) the paths in the range $x_{\mathrm{p}}=1500-2000 \mathrm{~km}$ (mode F2) that conventionally go in the same ionospheric region as the main path may be called similar. Yet, the paths in the range $x_{\mathrm{p}}=1500-2000 \mathrm{~km}$ along which the propagation mode (E mode) changes are not similar to the given path $x_{\mathrm{cr}}=1600 \mathrm{~km}$. The paths that pass through the main maximum of the F2-layer ionization and go out of the ionosphere are not similar to the given path either.

To test the diagnostic technique, we have examined two paths of the main source $x_{\mathrm{cr}}=1600 \mathrm{~km}$ and $x_{\mathrm{cr}}=1800$ $\mathrm{km}$ (F2 mode). As a secondary path we took a path $x_{\mathrm{p}}=1700 \mathrm{~km}$ long (F2 mode).

We selected the following parameters of the correlation ellipsoid with generalized properties of the field of random irregularities along the secondary path: $\mu^{2}=0.0004$ (it corresponds to the electron density perturbation of $2 \%$ of the background ionosphere), $a=10$ $\mathrm{km}, V=100 \mathrm{~m} / \mathrm{s}$. For these parameters from (20)-(22) we calculated variances of trajectory characteristics of a probe signal at an operating frequency $f=15 \mathrm{MHz}$. The resulting values were $\sigma_{\varphi p}^{2}=(90)^{2}$ (the standard deviation of the phase path $\left.\sigma_{\Phi p}=\frac{\sigma_{\varphi p} c}{2 \pi f}=286 \mathrm{~m}\right)$, $\sigma_{f \mathrm{p}}^{2}=0.04 \mathrm{~Hz}^{2} \quad\left(\sigma_{f} \mathrm{p}=0.2 \mathrm{~Hz}\right) ; \quad \sigma_{\Delta t \mathrm{p}}^{2}=2.04 \mu \mathrm{s}^{2}$ (the standard deviation of the group path $\sigma_{\Delta L \mathrm{p}}=c \sigma_{\Delta t \mathrm{p}}=428$ $\mathrm{m})$. These variances were used in (38)-(40) to determine the expected statistical characteristics of signals along paths of the main source. Integral coefficients in (38)-(40) were calculated through joint numerical integration system of equations for the main source $((17)$, (49)-(52), (55)-(57)) and for the test one ((17), (45)(48), (53), (54), (58)) with proper initial conditions. The values of statistical characteristics obtained are listed in Table 1.

We also considered the case when paths of the main source were $x_{\mathrm{cr}}=1600 \mathrm{~km}$ and $x_{\mathrm{cr}}=1700 \mathrm{~km}$, and the length of the secondary path $x_{\mathrm{p}}=1800 \mathrm{~km}$.

Table 1

Results of recalculation of statistical trajectory characteristics of the probe signal into characteristics of transmitted signals $\left(x_{\mathrm{p}}=1700 \mathrm{~km}, f=15 \mathrm{MHz}\right)$

\begin{tabular}{|c|c|c|c|}
\hline$x_{\mathrm{k}}, \mathrm{km}$ & $\sigma_{\Phi}, \mathrm{m}$ & $\sigma_{f}, \mathrm{~Hz}$ & $\sigma_{\Delta L}, \mathrm{~m}$ \\
\hline 1600 & 296 & 0.21 & 593 \\
\hline 1800 & 281 & 0.19 & 652 \\
\hline
\end{tabular}

The parameters of the correlation ellipsoid of irregularities on the secondary path are as follows: $\mu^{2}=0.0001$ (electron density perturbation of $1 \%$ of the background ionosphere), $a=20 \mathrm{~km}, V=150 \mathrm{~m} / \mathrm{s}$. Variances of trajectory characteristics of the probe signal calculated from (20)-(22) are $\sigma_{\varphi p}^{2}=(64)^{2} \quad\left(\sigma_{\Phi p}=203 \mathrm{~m}\right)$, $\sigma_{f \mathrm{p}}^{2}=0.0121 \mathrm{~Hz}^{2} \quad\left(\sigma_{f \mathrm{p}}=0.11 \mathrm{~Hz}\right), \quad \sigma_{\Delta t \mathrm{p}}^{2}=1.02 \mu \mathrm{s}^{2}$ $\left(\sigma_{\Delta L \mathrm{p}}=303 \mathrm{~m}\right)$.

Statistical moments of the probe signal and calculated integral coefficients (26)-(29), (41)- (44) were used in (38)-(40) to determine variances of trajectory characteristics of signals on the given paths. The calculation results are presented in Table 2.

Table 2

Statistical trajectory characteristics along given single-hop paths $\left(x_{\mathrm{p}}=1800 \mathrm{~km}, f=15 \mathrm{MHz}\right)$

\begin{tabular}{|c|c|c|c|}
\hline$x_{\mathrm{k}}, \mathrm{km}$ & $\sigma_{\Phi}, \mathrm{m}$ & $\sigma_{f}, \mathrm{~Hz}$ & $\sigma_{\Delta L}, \mathrm{~m}$ \\
\hline 1600 & 214 & 0.13 & 332 \\
\hline 1700 & 206 & 0.12 & 221 \\
\hline
\end{tabular}

From Tables 1 and 2 it follows that the calculated expected fluctuations of phase, Doppler frequency shifts, and group delay of signals along single-hop paths are consistent with known physical notions of scattering of decameter radio waves by ionospheric irregularities exceeding the size of the first Fresnel zone [Rytov et al., 1978]. Moreover, when the receiver of the main signal or the transmitter of the probe signal is located in the vicinity of focusing points of the field of the main source, the above relations require modification. At these points, the fun- 
damental solutions $\frac{\partial z_{0}}{\partial \beta_{\mathrm{n}}}, \frac{\partial z_{0}}{\partial \beta_{\mathrm{p}}}$ become zero [Kravtsov,

Orlov, 1980], and in Formulas (13), (14), (34), (35) features appear which are associated with ramification of solutions of boundary-value trajectory problems for the main and probe sources.

\section{CONCLUSION}

We have proposed a method for direct diagnostics of statistical trajectory characteristics of a decameter signal along an oblique ionospheric sounding path based on measurements of characteristics of a probe signal received by the transmitter of the main signal. The method is implemented using a model of the mean permittivity of the ionosphere. This model can be given by an analytical profile best matched to geophysical conditions. It is also allowed to use the advanced global models that represent the ionospheric electron density as discrete data. Applying the bicubic spline interpolation to discrete electron density profiles can provide continuity of first- and second-order derivatives required for calculations. The results of the numerical experiments have demonstrated efficiency of the mathematical apparatus in use to estimate the expected second-order statistical moments of phase, group delay, and Doppler frequency shift of a decameter signal along single-hop paths of mean length, when ground points of transmission and reception of main and probe signals are outside the vicinity of focusing points of the wave field. The proposed method for direct diagnostics of the stochastic radio channel can be used in the multifrequency mode, exclusive of the vicinity of the maximum usable frequency. To improve the quality of the diagnostics, a model of the anisotropic correlation ellipsoid of irregularities, which is oriented with respect to a radio path, can be adopted. Parameters of this ellipsoid can be determined from characteristics of a probe signal along the additional path, taking into account a priori information about generic properties of irregularities (e.g., their extension along geomagnetic field lines).

\section{REFERENCES}

Afanasiev N.T., Afanasiev A.N., Larunin O.A., Markov V.P. Phase fluctuations of radio waves experiencing total reflection from a randomly inhomogeneous plasma layer. J Atmos. SolarTerr Phys. 2010, vol. 72, no. 7-8, pp. 583-587. DOI: 10.1016/j.jastp.2010.02.014.

Afanasiev N.T., Laryunin O.A., Markov V.P. Phase fluctuations of a radio wave in the case of total internal reflection from a randomly inhomogeneous ionosphere. Radiophysics and Quantum Electronics. 2009, vol. 52, no. 10, pp. 699-704. DOI: 10.1007/s11141-010-9177-0.

Ageeva E.T., Afanasiev N.T., Baginov A.B., Kim D.B., Tanaev A.B., Chudaev S.O. Diagnostics of the state of the information channel using the statistical trajectory characteristics of the reference signal. Sovremennye naukoyemkie tekhnologii [Modern High Technologies]. 2020, no. 5, pp. 9-14. (In Russian).

Alimov V.A., Rakhlin A.V., Vybornov F.I. Model of interaction between decameter-decimenter radio waves and a strongly inhomogeneous mid-latitude ionosphere. Radiophysics and Quantum Electronics. 1997, vol. 40, no. 11, pp. 891-902.
Arnold V.I. Mathematical Methods of Classical Mechanics. Springer-Verlag, New York, 1978, 464 p.

Blagoveshchensky D.V. Short waves in anomalous radio channels: Experiment, simulation. Saarbrücken, LAP Lambert Academic Publ., 2011, 422 p.

Blagoveshchensky D.V., Zherebtsov G.A. Visokoshirotnye geofisicheskie yavleniya i prognosirovanie korotkovolnovykh radiokanalov [High-Latitude Geophysical Phenomena and the Prediction of Short-Wave Channels]. Moscow, Nauka Publ., 1987, 272 p. (In Russian).

Bova Yu.I., Kryukovsky A.S., Lukin D.S. Propagation of Frequency-Modulated Electromagnetic Radiation in the Earth's Ionosphere with Allowance for Absorption and the External Magnetic Field. J. Communications Technology and Electronics. 2019, vol. 64, no 1, pp. 1-12. DOI: 10.1134/S1064226919010030.

Bova K. Ionospheric Radio. Stevenage, United Kingdom. Institution of Engineering and Technology Publ., 1990, 600 p.

Davies K. Ionospheric Radio. Stevenage, United Kingdom. Institution of Engineering and Technology Publ., 1990, 600 p.

Gershman B.N., Erukhimov L.M., Yashin Yu.Ya. Volnovye yavleniya $v$ ionosphere $i$ kosmicheskoi plazme [Wave Phenomena in the Ionosphere and Space Plasma]. Moscow, Nauka Publ., 1984, 392 p. (In Russian).

Gusev V.D., Ovchinnikova N.P. Model definition of volumetric characteristics of ionosphere non-uniformities. Geomagnetism and Aeronomy. 1980, vol. 20, no 4, pp. 434-437.

Ipatov E.B., Kryukovskii A.S., Lukin D.S., Palkin E.A., Rastyagaev D.V. Methods of simulation of electromagnetic wave propagation in the ionosphere with allowance for the distributions of the electron concentration and the Earth's magnetic field. J. Communications Technology and Electronics. 2014, vol. 59, no 12, pp. 1341-1348. DOI: 10.1134/S1064 226914120079.

Ishimaru A. Wave Propagation and Scattering in Random Media. John Wiley \& Sons, 1999, 604 p.

Kazantsev A.N., Lukin D.S., Spiridonov Yu.G. A method for studying the propagation of radio waves in an inhomogeneous magnetoactive ionosphere. Space Res. 1967, vol. 5, iss. 4, pp. 593-600.

Klyatskin V.I. Stokhasticheskie uravneniya [Stochastic Equations]. Moscow, Fizmatlit Publ., 2008, vol. 1, 317 p. (In Russian).

Kravtsov Yu.A., Orlov Yu.I. Geometrical Optics of Inhomogeneous Medium. Berlin, Springer-Verlag Publ., 1990, 312 p.

Kryukovskii A.S., Lukin D.S., Kir'yanova K.S. Method of extended bicharacteristic system in simulating wave propagation in ionospheric plasma. J. Communications Technology and Electronics. 2012, vol. 57, no. 9, pp. 1039-1045. DOI: 10.1134/S1064226912080177.

Kryukovsky A.S., Kurkin V.I., Laryunin O.A., Lukin D.S., Podlesnyi A.V., Rastyagaev D.V., Chernyak Y.M. Numerical modeling of amplitude maps for the corrected IRI-2012 model with smooth ionospheric disturbances. J. Communications Technology and Electronics. 2016, vol. 61, no 8, pp. 920-925. DOI: $10.7868 / \mathrm{S} 0033849416080118$.

Kurkin V.I., Nosov V.E., Ponomarchuk S.N., Savkov S.S., Chistyakova L.V. Method for operative diagnostics of HF radio channel. Issledovaniya po geomagnetizmu, aeronomii $i$ fizike Solntsa [Res. on Geomagnetism, Aeronomy and Solar Physics]. Novosibirsk, Nauka Publ., 1993, vol. 100, pp. 168188. (In Russian).

Mitra S.K. The Upper Atmosphere. Calcutta, Royal Asiatic Society of Bengal Publ., 1947, 616 p.

Rawer K. Wave Propagation in the Ionosphere. Dordrecht, Springer Netherlands Publ., 1993, 479 p.

Rytov S.M., Kravtcov Yu.A., Tatarsky V.I. Vvedenie $v$ statisticheskuyu radiofisiku. Chast' 2. Sluchainye polya. (Introduction to Statistical Radiophysics. Part 2: Random Fields). Moscow, Nauka Publ., 1978, p. 464. (In Russian). 
Tereshchenko V.D. To the question of the influence of horizontal gradients of electron concentration on the MUF and the trajectory of radio wave propagation in the ionosphere. Morfologiya i fizika polyarnoi ionosfery [Morphology and Physics of the Polar Ionosphere]. Leningrad: Nauka Publ., 1971, pp. 228-235. (In Russian).

Vologdin A.G., Vlasova O.K., Prikhod'ko L.I. Fluctuations of the group path and group-delay time of waves obliquely reflected by a plane-layered medium. Radio Engineering and Electronics. 2007, vol. 52, no. 10, pp. 1100-1103.
Vologdin A.G., Prikhod'ko L.I., Shirokov I.A. Statistics of the Doppler frequency shift of radio waves reflected from the parabolic ionospheric layer. V Vserossiiskie Armandovskie chteniya "Radiofizicheskie metody $v$ distantsionnom zondirovanii sred: Doklady [Proc. V All-Russian Armandov Readings "Radiophysical Methods in Remote Sensing of Media"]. Murom, 2012, pp. 159-163. (In Russian).

How to cite this article

Afanasiev N.T., Chudaev S.O. Diagnostics of the stochastic ionospheric channel in the decameter band of radio waves. Solar-Terrestrial Physics. 2020. Vol. 6. Iss. 4. P. 66-73. DOI: 10.12737/stp-64202010. 